\title{
WiFi Fingerprinting Localization for Intelligent Vehicles in Car Park
}

\author{
Dinh-Van nguyen, Raoul de Charette, Fawzi Nashashibi \\ RITS Team \\ INRIA Paris \\ Paris, France \\ Dinh-van.nguyen@inria.fr
}

\author{
Trung-Kien Dao, Eric Castelli \\ MICA Institute (HUST - Grenoble INP) \\ Hanoi University of Science and Technology \\ Hanoi, Vietnam \\ Trung-Kien.Dao@mica.edu.vn
}

\begin{abstract}
In this paper, a novel method of WiFi fingerprinting for localizing intelligent vehicles in GPS-denied area, such as car parks, is proposed. Although the method itself is a popular approach for indoor localization application, adapting it to the speed of vehicles requires different treatment. By deploying an ensemble neural network for fingerprinting classification, the method shows a reasonable localization precision at car park speed. Furthermore, a Gaussian Mixture Model (GMM) Particle Filter is applied to increase localization frequency as well as accuracy. Experiments show promising results with average localization error of $0.6 \mathrm{~m}$.
\end{abstract}

Keywords-component; Intelligent Vehicles; Indoor localization; WiFi Fingerprinting; Ensemble Neural Network; Particle Filter; Sensor Fusion;

\section{INTRODUCTION}

As intelligent vehicles become closer to reality, a vast majority of researching resources are focusing on outdoor localization where GNSS is available. Works on indoor scenarios as well as the transition between indoor and outdoor are not yet fully addressed while a report shows that vehicles spend $95 \%$ of the time in car parks [1]. Moreover, a study in France suggests a high average searching time for a free slot in car parks from $10-40$ minutes in major cities which results in 70 million hours or 700 million euro cost a year [2]. This indicates a big gap to fill for intelligent vehicles localization.

Due to lack of space in urban cities, more and more multistory car parks are built to meet drivers' need. Inside these car parks, there are certain constraints about vehicles localization such as:

- No GPS signal.

- Average speed in a car park is around $10-12 \mathrm{~km} / \mathrm{h}[3]$.

- Areas with identical physical features.

Normally, a localization system for an intelligent vehicle is a fusion of multiple sensors such as: GPS antenna, LiDAR, camera, Inertial Measurement Unit (IMU), etc. Among them, the GPS antenna is critical for two primary roles: a global coordinate reference for fusion of multiple sensors (each with its own local coordinate) and a semantic indicator for a vehicle to perceive the surrounding environment. Thus, the lack of GPS signal for the indoor environment such car parks will most likely interrupt the vehicle localization system. Consequently, an alternative of GPS signal for indoor environment is required.

Speed is one of the keys constraints to the indoor localization of vehicles also. Although sensors like camera, LiDAR, IMU, etc. are doing well at much higher speed, those sensors do not provide a replacement for GPS signal or require a complex and expensive map of the environment to function. Other solutions for indoor movement tracking including beacons, WiFi signal, Ultra-Wideband, etc. often operate at low sampling frequency hence not suitable for the speed of a vehicle.

Another constraint mentioned above is the symmetrically identical structure of a car park. This characteristic of car parks increases the uncertainty of localization methods which use physical features such as laser/visual SLAM, image processing, etc. causing them to converge slower to true position. Also, symmetricity will add more confusion to the kidnapped robot problem [4]. This further emphasizes the need for a global positioning system that can give absolute positioning feedback to the vehicle.

This paper will address the issue of car park localization and the transition between the car park and outdoor environment. By introducing a bagging ensemble neural network and a hybrid offline phase learning to $\mathrm{WiFi}$ fingerprinting localization method, the method is capable of replacing GPS signal for the indoor environment localization. Also, a fusion with IMU using Gaussian Mixture Model Particle Filter will be presented to increase sampling frequency as well as accuracy of the whole system.

The paper is organized as follows: Section I gives a brief introduction of the problem and the idea for a solution. Section II summarizes state of the art of car park localization for vehicles. Section III explains WiFi Fingerprinting method as well as adaptation to vehicles localization. Section IV presents a Gaussian Mixture Model Particle Filter to fuse information from IMU and WiFi Fingerprinting method to increase sampling frequency and accuracy of localization. Section $\mathrm{V}$ gives details about experiment setup and results.

\section{STATE OF THE ART}

In the last few years, research community in Intelligent Vehicles has been developing several dedicated systems for car park localization and navigation. These systems can be 
categorized into two major classes: an absolute positioning approach with a pre-built map of the environment and a relative positioning approach with local feature detection.

In absolute positioning approach, two conditions are assumed: a pre-built map of the environment is available and little to no change in the environment static obstacles. This method often makes use of vision sensors such as LiDAR or camera. Work from [5] divides a map into known static objects like walls, parking slots, etc. and semi-static objects like parked cars. Instead of performing a full SLAM problem, a particle filter for localization on a pre-calculated probability grid map is deployed. A full SLAM approach can be found in [6] with RaoBlackwellized particle filter SLAM together with a static map of the environment. The idea is to reduce the uncertainty of the SLAM with a known static map thus improving the accuracy of localization. Instead of mounting LiDAR sensor on vehicles, the approach in [7] proposes to embed LiDAR sensors in the environment. Using RANSAC algorithm, the authors have successfully localized vehicles within $8 \mathrm{~cm}$ of mean errors. A camera approach in this category is found in [8]. The method builds a detailed 3D map of the environment together with dead reckoning algorithm and particle filter to localize vehicles inside a car park. This method also requires a GPS position initialization for the 3D matching process. In general, methods fall into this category often perform well for a carefully mapped environment. However, these methods are expensive in computation as well as in map building process and sometimes also costly sensors. They are dedicated to one environment without any or little consideration of transition phase for the system from indoor to outdoor or vice-versa.

For relative positioning approach, vehicles are located relatively to surrounding local objects such as other vehicles, lane marking, parking lot positions etc.. An example of this method can be found in [9] with lane extraction and relative positioning. A vehicle to vehicle communication method to improve localization accuracy is proposed in [10]. A method for detecting parking slot position in [11] using multiples cameras on vehicles yields 0.2 meters of error in localization. One notable characteristic of this approach is the uncertainty of relative positioning increase significantly as the uncertainty of each agent adds up. Also, this method suffers heavily from accumulated error as there is little chance to do correction once error is made.

\section{ENSEMBLE BAGGING WIFI FINGERPRINTING}

WiFi Fingerprinting localization is a popular method for indoor localization typically for pedestrian walking. The method makes use of existing WiFi infrastructure (WiFi Access Points - APs, WiFi receiver) to determine location based on an offline mapping phase. The main argument of this method is that the combination of WiFi signal strengths from multiple static APs in the environment for one position is unique. By learning these unique features for several key positions in the environment, one can estimate its location just by scanning WiFi signal strength. Generally, WiFi Fingerprinting has two phases: offline phase and online phase.

Offline phase of WiFi Fingerprinting method is where signal strengths from multiple locations are gathered and stored with

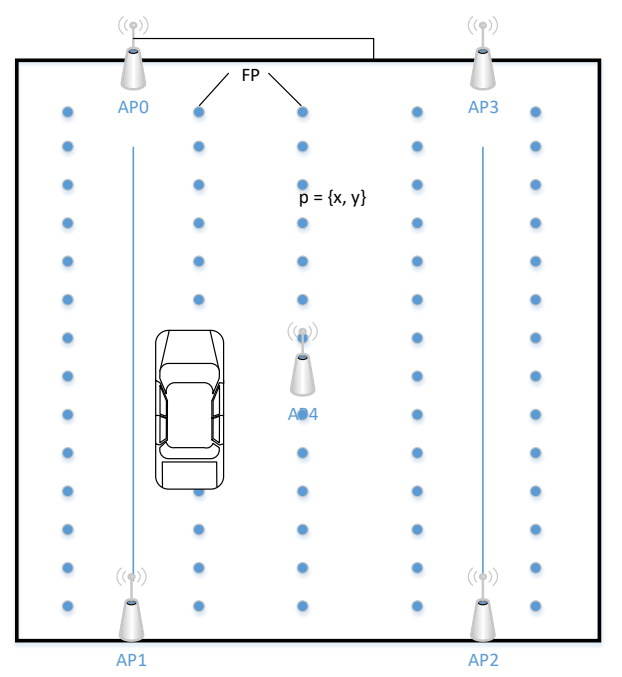

Fig. 1. Illustration of offline phases in WiFi Fingerprinting

corresponding to coordinate of each location. As shown in Fig. 1 , each blue dot will be called a fingerprint (FP). For each fingerprint, a mapping of scanned WiFi signal strength and its coordinate is stored.

$$
\left\{X_{i}, \rho_{l}\right\}=\left\{x_{i, 1}, x_{i, 2}, x_{i, 3}, \ldots, x_{i, n}, \rho_{l}\right\}
$$

where $x_{i, j}$ is WiFi signal strength from $\mathrm{jth} \mathrm{WiFi}$ APs that is recorded from the absolute coordinate $\rho_{l}$ of $F P_{l}$ in ith scan. A collection of multiple scans from each FP in this offline phase will act as a reference for estimating position in online phase. One advantage of this method is the coordinate of fingerprint location can be measured in global frame (i.e. GPS frame) so that the transition from the indoor to the outdoor environment can be done seamlessly. At the same time, this method does not suffer from symmetricity of car park and highly feasible and scalable due to availability of APs in the environment.

In the online phase where localization estimation is carried out, the vehicle will move inside the environment while scanning for WiFi signal strength from surrounding APs. A likelihood function based on data from offline phase is defined as:

$$
h(X)=\gamma_{\rho_{l}}
$$

Here, $X$ is input signal scan and $\gamma_{\rho_{l}}$ is the likelihood score of $X$ to be scanned at $F P_{l}$ in the environment with regard to offline collected mapping coordinate $\rho_{l}$. In most of the cases, the position with the highest likelihood score will be the output of localization process.

WiFi Fingerprinting appears to perform well for a human user in multiple indoor scenarios with modification in either hardware or software. A $20-40 \mathrm{~cm}$ of error are reported in [12][14]. However, there are several issues with this method when it comes to vehicles movement:

- Low sampling frequency: the time to complete a scan of $\mathrm{WiFi}$ signals in a particular environment depends on multiple factors but generally falls into 1 second range $(1 \mathrm{~Hz})$. This sampling frequency is neglected with human 
walking speed around $1.4 \mathrm{~m} / \mathrm{s}$ - although in practice, most systems require user to walk at a lower speed for a better precision. In contrast, a vehicles moving in a car park is expected to move at $3-3.3 \mathrm{~m} / \mathrm{s}$ [3]. This results in a single measurement every 3 meters. In addition, localization system for a vehicle is much more demanding in term of precision and sampling frequency. Consequently, a classic WiFi fingerprinting system alone is inadequate for vehicle localization.

- Due to the high speed movement of vehicles, the signal scan of $\mathrm{WiFi}$ is expected to have higher variance and noise. In fact, with one measurement every 3 meters, the signal scan vector does not reflect any single position characteristic but rather a range.

To solve these issues, changes in both offline phase and online phases are proposed. A hybrid learning database in offline phase and an ensemble neural network for online phase is implemented as follows.

\section{A. Hybrid Offline Phase}

In classical approach of WiFi Fingerprinting localization method, offline collection of mapping between position (Fingerprint - FP) and Received Signal Strength Indicator (RSSI) from Access Points (APs) in the environment is done statically. This means at each FP, a number of scans for RSSI are performed and stored to model the signal strength feature of that particular position. However, as suggested above, this method likely produces biased observation of signal strength as vehicles moving at much faster speed thus results in a range of signal scans instead of static position signal scan. We then define a scan range as the distance traveled by a vehicle from initiation to complete of a WiFi signal scan.

Consider a typical scan of RSSI in online phases as in Fig. 2. For 1 scan of signal strength at $1 \mathrm{~Hz}$ sampling frequency, the vehicle is likely to pass by a Fingerprint defined in offline phase. In the best case, the vehicle is likely to travel $3.3 \mathrm{~m}$ (at $3.3 \mathrm{~m} / \mathrm{s}$ ), passing by the fingerprint at exact centre of the path as shown in the figure. This is a significant range that cannot be represented with only static scans at fingerprint position in offline phase.

One way to address this issue is to include dynamic signal scans in offline phase to emulate the character of a real-time online phase observation. Instead of only collecting scan statically at fingerprint position in offline phase, the vehicle will also move at different speeds passing by fingerprint positions. Multiple scans within a representative area around a fingerprint will also be recorded.

$$
\left\{X_{i}, \rho_{l}\right\}=\left\{x_{i, 1}, x_{i, 2}, x_{i, 3}, \ldots, x_{i, n}, \rho_{l}^{\prime}\right\}
$$

Where

$$
\rho_{l}^{\prime} \in(\rho-\Delta \rho, \rho+\Delta \rho)
$$

The representative area for a fingerprint position is defined as in (4). Note that, as in (3), although the real position $\rho^{\prime}$ that is mapped with the scan is different from fingerprint position $\rho$, the final mapping will still be the static fingerprint position to preserve fingerprinting characteristic. Thus all scans collected

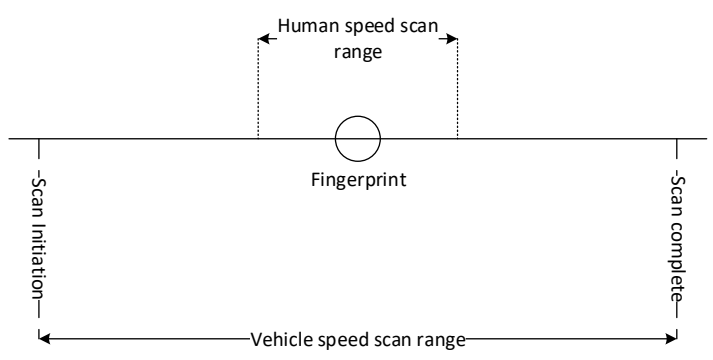

Fig. 2. Scan range of vehicles in online phase.

within the representative area of a fingerprint will be mapped to the fingerprint position only.

$$
\Delta \rho=\bar{v}_{\text {vehicle }} / 2
$$

The fingerprint representative area parameter $\Delta \rho$ will be determined as in (5) with average speed $\bar{v}_{\text {vehicle }}$ since in the best case scenario, the vehicle will pass by the fingerprint location at exactly the middle of scanning. One reason for that is if worst case scenario where the vehicle passed by the fingerprint location at either end of the scanning cycle were considered, then the representative area would be much wider and therefore would introduce more noise to the collected data. The worst case scenario also did not reflect the desired feature for targeted fingerprint location. In case of the car park with an average speed of a vehicle is $3.3 \mathrm{~m} / \mathrm{s}, \Delta \rho$ is approximately $1.6 \mathrm{~m}$. This closely brings back the problem to human walking speed if we consider the representative area as a fingerprint.

Finally, a normalization of collected RSSI in each scan is performed to reduce the numerical impact of signal strength feature. A detected AP RSSI would be normalized in the range $[0,1)$ with 0 as the weakest possible signal strength and 1 as the strongest possible signal strength.

$$
x_{i}=\left\{\begin{aligned}
&-1, A P_{i} \text { undetected } \\
& 1-\frac{(-1) \times R S S I}{100}, A P_{i} \text { detected }
\end{aligned}\right.
$$

\section{B. Ensemble Neural Network with Bagging}

In online phase, a likelihood function is designed to estimate which fingerprinting is closest to input scanning signals. There are several algorithms to estimate likelihood score of input scanning signal. In this paper, an Ensemble Neural Network with Bagging is proposed to be the classifier. This method is known to perform well with high variance and noisy data. In this case, the WiFi signal strength collected during online phase is noisy with high variance as suggested in the previous section.

Ensemble neural network with bagging (Bootstrap Aggregating) is a method of combining multiple learning models to derive better prediction results[15], [16]. Consider a mapping $\{X, Y\}$ where $X$ is a vector of features and $C$ is corresponding class. In this paper, $X$ is a vector of signal strengths scanned at a particular position in the environment and $Y$ is the label of FP position defined in offline phase. A function estimator which results from a set of training samples and a classification model is formed. 
2018 International Conference on Indoor Positioning and Indoor Navigation (IPIN), 24-27 September 2018, Nantes, France

$$
\left.g(\cdot)=h\left(\left(X_{1}, Y_{1}\right),\left(X_{2}, Y_{2}\right), \ldots,\left(X_{n}, Y_{m}\right)\right)\right)
$$

Bagging algorithm is executed as follows:

- Construct bootstrap sample by randomly sampling with replacement $n$ times from original training data. Let $X^{\wedge}{ }_{i}$ and $Y^{\wedge}{ }_{i}$ is a bootstrap sample data from collected database $\{X, Y\}$ :

$$
\left(X_{1}^{\wedge}, Y_{1}^{\wedge}\right),\left(X_{2}^{\wedge}, Y_{2}^{\wedge}\right), \ldots,\left(X_{n}^{\wedge}, Y_{m}^{\wedge}\right)
$$

- Compute bootstrap estimator $g^{\wedge}(\cdot)$ by applying same classification model to newly formed bootstrap sample:

$$
\left.g^{\wedge}(\cdot)=h\left(\left(X_{1}^{\wedge}, Y_{1}^{\wedge}\right),\left(X_{2}^{\wedge}, Y_{2}^{\wedge}\right), \ldots,\left(X^{\wedge}{ }_{n}, Y_{m}^{\wedge}\right)\right)\right)
$$

- Repeat two steps above for $\mathrm{K}$ times with $\mathrm{K}$ is large the bagging estimator is:

$$
g_{\text {bagg }}^{\wedge}(\cdot)=\frac{1}{K}\left(\sum_{i=1}^{K} g^{\wedge}(\cdot)\right)
$$

Theoretically, as $\mathrm{K}$ goes to infinity, the estimator converges to the expected value. In practice, a finite large $\mathrm{K}$ is expected to improve Monte Carlo accuracy.

Since vehicles are running at different speed, scanned RSSI is expected to be noisy and high variance. Ensemble Neural Network appears to be functioning well with high variance and noisy data. By picking randomly with replacement training data from whole dataset, the combination of final learning models is able to overcome bias and eliminate noise.

\section{GuAsSian MiXture Model Particle FILTER}

As mentioned in previous section, WiFi fingerprinting localization suffers from low sampling frequency. To enhance the performance, a fusion with other sensors information is recommended. In this paper, a Gaussian mixture model particle filter is deployed to fuse WiFi fingerprinting localization with other sensor such as IMU information. The fusion strategy is illustrated in Fig. 3.

Given a signal scan, a classification result from the ensemble neural network is defined as a list of fingerprints and their corresponding confidence $\left\{F P_{i}, C_{i}\right\}$. In classic fingerprint solution, it is likely that the highest confidence fingerprint will be the final prediction of the method. However, due to environment noise and multipath effect of signal transmission, the highest confidence fingerprint does not always represent the best estimation. One way to improve the prediction accuracy is to consider few fingerprints with highest confidence.

A particle filter with GMM model is established as following:

- Initialization: The filter is initialized after the first frame of WiFi Fingerprinting. Particles are generated around the highest confidence fingerprint that results from the ensemble neural network with a standard deviation $\sigma$. ( $\sigma$ is estimated from the statistic of WiFi fingerprinting localization experiments).

- Updating transition: A constant speed model that receives velocity and heading angle of the vehicle as inputs. This model moves all particles with an assumption that at $10 \mathrm{~Hz}$ frequency of IMU, movement of a vehicle can be considered as constant speed.

- Predicting transition: Top 3 highest confidence FPs returned from network will be considered as possible observation. Score of each particle will be updated as in (11). Here, $x_{t}$ is a particle at time $\mathrm{t}, c_{i}$ is the confidence of fingerprint ith returned by ensemble neural network, $\sigma_{i}$ is standard deviation of observation at fingerprint ith and $\mu_{i}$ is coordinate of Fingerprint ith.

$$
\operatorname{GMM}\left(x_{t}\right)=\sum_{i} c_{i} P\left(x_{t} \mid \sigma_{i}, \mu_{i}\right)
$$

A Gaussian distribution is assumed with multiple observations are considered. Each observation has a posteriori confidence score. The higher the confidence, the smaller variance from mean is expected. Thus, given an estimated standard deviation $\sigma$ for $\mathrm{WiFi}$ fingerprinting localization observation, the standard deviation for each fingerprinting observation in this case will be calculated as:

$$
\sigma_{i}=1-c_{i} \sigma
$$

Since WiFi scan frequency goes at $1 \mathrm{~Hz}$ and IMU data is filtered at $10 \mathrm{~Hz}$, there are cases where there is no observation is fed into particle filter except IMU information. At these cases, all particles will be updated with constant speed model with equal score. Final result of particle filter estimation is weighted sum of all particles with its score.

$$
x=\frac{1}{n} \sum_{t} x_{t} \sum_{i} c_{i} \frac{1}{\sqrt{2 \pi\left(1-c_{i} \sigma\right)^{2}}} e^{-\left(x_{t}-\mu_{i}\right)^{2}} / 2\left(1-c_{i} \sigma\right)^{2}
$$

Note that by talking weighted sum of all particles instead of just the highest score particle as the final prediction, this allows room for recovery from biased observation. After receiving predicted result, a random proportion of particles is sampled with replacement using multinomial resampling method.

\section{EXPERIMENTS AND RESULTS}

During nearly one year period, experiments have been carried out in Rocquencourt campus - INRIA Paris. The setup of experiments is explained as follows.

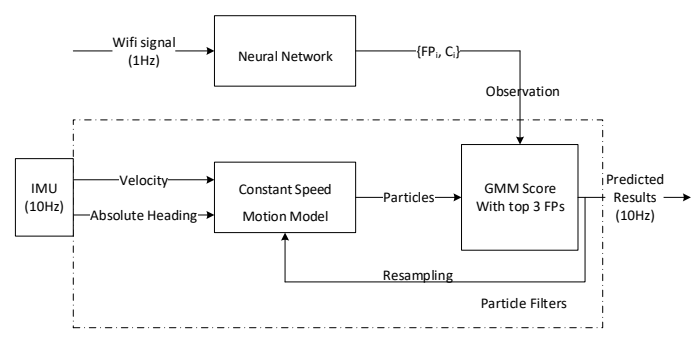

Fig. 3. Fusion of IMU and WiFi using GMM Particle Filter

There are two testing platforms: a Cybercars (blue) and an electric car Citroën C1 (red) (Fig. 4). The Cybercar is used in both online and offline phase of WiFi fingerprinting localization. The $\mathrm{C} 1$ is used in testing phase only. Sensors on both vehicles are:

- RTK GPS antenna 
- Standard Micro-Next USB WiFi receiver

- IMU, gyroscope

- Front and back LiDAR sensors.

All experiments are performed in the outdoor environment, near parking lot and surrounded by buildings (instead of an indoor car park). The reason for adopting the outdoor scenario is to take advantage of centimetre precision RTK GPS as the ground truth for experiments' results evaluation later. The area of experiments is shown in Fig. 5.

In the offline phase of WiFi Fingerprinting method, the Cybercar collected static RSSI scan at fingerprints position which was chosen to be at roughly every $5 \mathrm{~m}$ apart on the travelling route. Dynamic scans were collected in next 3 days, after static scans, and at some random times of the day.

In the online phase, both Cybercar and $\mathrm{C} 1$ are running tests on two localization modes: only WiFi Fingerprinting and fusion of WiFi and IMU using GMM Particle Filter. Both car are moving within $2.5-3.5 \mathrm{~m} / \mathrm{s}$ of average speed in all experiments to comply with the average speed of vehicles moving inside a car park.

For WiFi only localization method using fingerprinting, results obtained were shown in [17]. Within $1012.9 \mathrm{~m}$ of the travel path, the maximum error recorded is $6.84 \mathrm{~m}$, and the average error is $2.25 \mathrm{~m}$. However, the sampling frequency of this method is restricted to the sampling frequency of WiFi scan which is $1 \mathrm{~Hz}$.

By fusing this result with IMU information using GMM Particle Filter, the localization performance is drastically improved. Fig. 5 shows a smoother and much more accurate localization outcome of a single test run is obtained in comparision to the result of $\mathrm{WiFi}$ fingerprinting method alone. With GMM fusion, the localization system is capable of running at $10 \mathrm{~Hz}$ frequency instead of just $1 \mathrm{~Hz}$. Not only that, the error of localization is significantly reduced. Having statistic from $\mathrm{WiFi}$ fingerprinting localization results in [17], a standard deviation $\sigma=1.5$ is used for the fusion.

From March 2017 to January 2018, 64 test runs were recorded with Cybercar and $\mathrm{C} 1$. Fig. 6a shows a single run statistic with maximum error is $1.223 \mathrm{~m}$ and mean error is $0.473 \mathrm{~m}$. The mean error estimation and variance of each experiment error are evaluated. A portion of those data is presented in Fig. 6b. For all experiments, the maximum mean error is $0.62 \mathrm{~m}$ and the maximum variance is 0.175 . This statistic shows the stability of the method over the course of one-year experiments. Note that, during this time, there was no significant change in the WiFi infrastructure and the environment structure. We also targeted only infrastructure mode WIFI networks in the area to reduce noise in the signal collection process.

In addition, with a random initial position, there are cases where the first position of the vehicle is not within the range of any fingerprint. These cases are examined and results are shown in Fig. 7a. Here, due to the initial position, the maximum error is large at $3.28 \mathrm{~m}$. However, after 23 frames, the particle filter quickly converges to reduce the error by $47 \%$ to reach $1.722 \mathrm{~m}$ of maximum error. This happens due to the observation from
WiFi fingerprinting localization helps converging the particle filter. Once a correct observation is made, the bias from starting position is quickly reduced. The results also prove that WiFi GMM fusion plays a critical role in correcting bias and accumulated error from the IMU motion model. The mean error for the entire path is recorded at $0.77 \mathrm{~m}$. The cumulative distribution of error for this case is shown in Fig. 7b. At $\sigma=$ $1.5,95.3 \%$ of error estimations are below one sigma.

Furthermore, a distribution of all error estimations for every conducted experiments is shown in Fig. 8a. The distribution confirms the observation made from Fig. 6b with most of the error estimations are around $0.5 \mathrm{~m}$. The cumulative distribution of these estimation demonstrates similar result in Fig. 8b. With $\sigma$ is estimated around $1.5,99.3 \%$ of the error estimation from all experiments is below one sigma. This suggests a reliable prediction in localization process of the system in general.

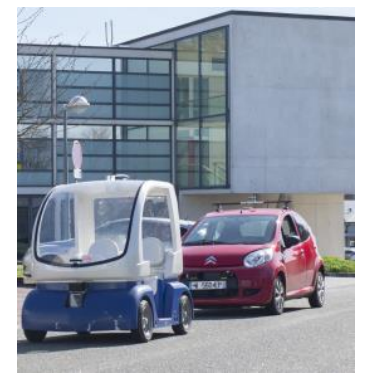

Fig. 4. Testing platforms: Cybercar (blue) and Citroen C1 (red)

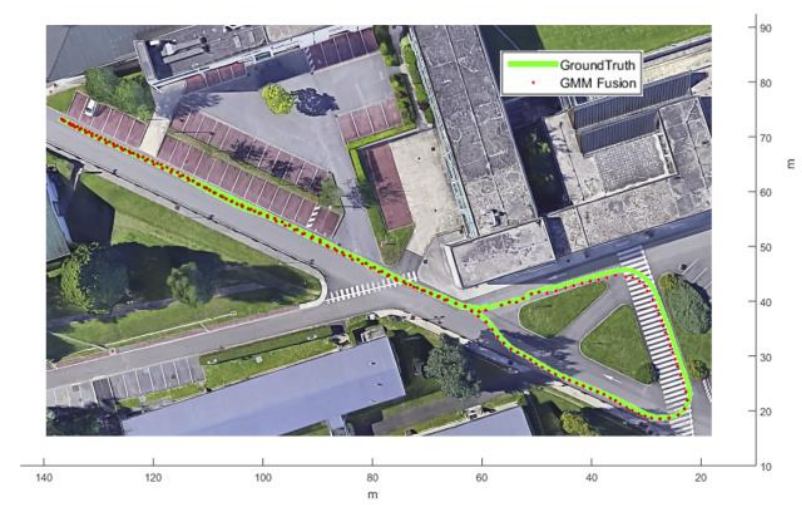

Fig. 5. GMM fusion localization result of a single run in INRIA Rocquencourt
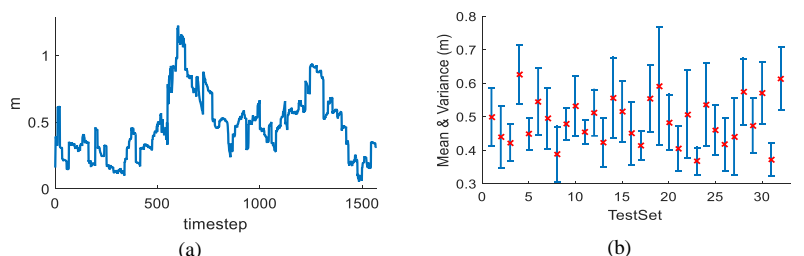

(b)

Fig. 6. (a) Single run of GMM Fusion error estimation; (b) Mean \& Variance of localization error in random representative test runs. 


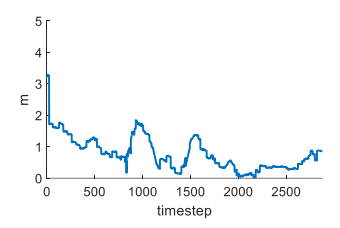

(a)

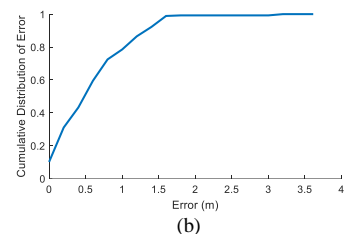

(b)
Fig. 7. (a) Single run with initial position out of fingerprint range. (b) cumulative distribution of error.

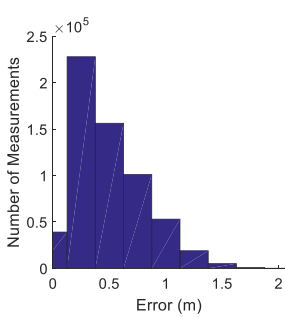

(a)

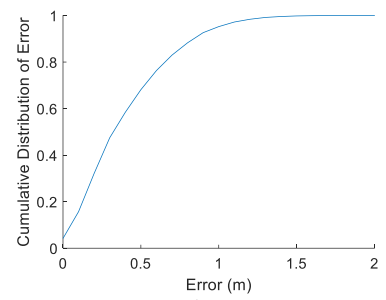

Fig. 8. (a) Distribution of error estimation in all test runs; (b) Cumulative distribution of error for all test runs.

\section{CONCLUSION}

In this paper, a novel method of localizing intelligent vehicles in a GPS-denied environment such as car park is proposed. A number of constraints are identified such as lack of GPS, vehicle speed and symmetricity of the environment. The proposed method has two main components: (1) an ensemble neural network for WiFi fingerprinting localization and (2) a Gaussian Mixture Model Particle Filter to fuse WiFi fingerprinting with IMU information.

In the first component, by applying a hybrid offline phase for data collection and introducing an ensemble neural network, localization results with $2.25 \mathrm{~m}$ mean error were obtained. By mapping signal scan at fingerprinting location to GPS frame coordinate, it also allows seamless transition between environments.

The second component provides improvements for localization system in both sampling frequency and accuracy. By deploying a Gaussian mixture model into a bootstrap Particle Filter, it allows the system to overcome WiFi fingerprinting limitations.

Experiments in the span of nearly a year from March 2017 to January 2018 shows promising results of localization for intelligent vehicles. A $99.3 \%$ of error estimation is under one sigma (with $\sigma=1.5$ ). Mean error of localization is $0.62 \mathrm{~m}$ with low standard variation of 0.175 . These results prove the correctness and stability of the proposed system in a long run.

Still, there are several key factors which need to be addressed in this work such as: a real multistory carpark situation for experiments or changes in the environment condition (i.e. weather, physical changes, etc.). The experiments in this work did not address z-axis of the localization problem (for multistory carpark case) and there was no experiment conducted under extreme weather condition due to the nature of RTK GPS ground truth. Also, no changes in physical condition such as new structure, changes in APs location was recorded. These are factors which could potentially hurt the performance of the proposed system. In the future, these factors will be tackled.

\section{REFERENCES}

[1] P.Barter, "Cars are parked 95\% of the time," (February 22, 2013) Retrieved from https://www.reinventingparking.org/2013/02/cars-areparked-95-of-time-lets-check.html

[2] E. Gantelet and A. Lefauconnier, "The time looking for a parking space: Strategies, associated nuisances and stakes of parking management in France," pp. 1-7, 2006.

[3] S. Belloche, "On-street Parking Search Time Modelling and Validation with Survey-based Data," Transp. Res. Procedia, vol. 6, no. June 2014, pp. 313-324, 2015.

[4] S. P. Engelson and D. V. McDermott, "Error correction in mobile robot map learning," Proc. 1992 IEEE Int. Conf. Robot. Autom., pp. 2555-2560, 1992.

[5] B. H. Groh, M. Friedl, A. G. Linarth, and E. Angelopoulou, "Advanced Real-time Indoor Parking Localization based on Semi-Static Objects," Inf. Fusion (FUSION), 2014 17th Int. Conf., pp. 1-7, 2014.

[6] S. Wahl, P. Schlumberger, R. Rojas, and M. Stampfle, "Localization inside a populated parking garage by using particle filters with a map of the static environment," IEEE Intell. Veh. Symp. Proc., vol. 2015-Augus, no. Iv, pp. 95-100, 2015.

[7] A. Ibisch, S. Stumper, H. Altinger, M. Neuhausen, M. Tschentscher, M. Schlipsing, J. Salinen, and A. Knoll, "Towards autonomous driving in a parking garage: Vehicle localization and tracking using environmentembedded LIDAR sensors," IEEE Intell. Veh. Symp. Proc., no. Iv, pp. 829-834, 2013.

[8] J. Bojja, M. Kirkko-Jaakkola, J. Collin, and J. Takala, "Indoor Localization Methods Using Dead Reckoning and 3D Map Matching," $J$. Signal Process. Syst., pp. 1-12, 2013.

[9] J. Levinson, M. Montemerlo, and S. Thrun, "Map-Based Precision Vehicle Localization in Urban Environments," Robot. Sci. Syst. III, pp. 121-128, 2008.

[10] A. Amini, R. M. Vaghefi, J. M. De Garza, and R. M. Buehrer, "Improving GPS-Based Vehicle Positioning for Intelligent Transportation Systems," no. Iv, pp. 1023-1029, 2014.

[11] S. Houben, M. Komar, A. Hohm, S. Luke, M. Neuhausen, and M. Schlipsing, "On-Vehicle Video-Based Parking Lot Recognition with Fisheye Optics,” 2013 16th Int. Ieee Conf. Intell. Transp. Syst. -, no. Itsc, pp. 7-12, 2013.

[12] M. Kotaru, K. Joshi, D. Bharadia, and S. Katti, "SpotFi : Decimeter Level Localization Using WiFi," Sigcomm 2015, pp. 269-282, 2015.

[13] J. Xiong and K. Jamieson, "ArrayTrack: A Fine-Grained Indoor Location System,” USENIX Symp. Networked Syst. Des. Implement., no. 279976, pp. 71-84, 2013.

[14] S. Kumar, S. Gil, D. Katabi, and D. Rus, "Accurate indoor localization with zero start-up cost," Proc. 20th Annu. Int. Conf. Mob. Comput. Netw. - MobiCom '14, pp. 483-494, 2014.

[15] L. Breiman, "Bagging Predictors," Mach. Learn., vol. 24, pp. 123-140, 1996.

[16] T. G. Dietterich, "Ensem ble Methods in Mac hine Learning," Mult. Classif. Syst., vol. 1857, pp. 1-15, 2000.

[17] N. Dinh-van and F. Nashashibi, "Indoor Intelligent Vehicle localization using WiFi Received Signal Strength Indicator," no. Icmim, pp. 1-4, 2017 . 